\title{
Mikrowellenspektrum, Struktur, Dipolmoment und internes Hinderungspotential von Dimethyldisulfid
}

\author{
D. Sutter, H. Dreizler und H. D. Rudolph \\ Physikalisches Institut der Universität Freiburg i. Br. \\ (Z. Naturforschg. 20 a, 1676-1681 [1965]; eingegangen am 13. September 1965)
}

\begin{abstract}
The microwave spectra of $\mathrm{CD}_{3}-\mathrm{S}-\mathrm{S}-\mathrm{CD}_{3}$ and $\mathrm{CH}_{3}-\mathrm{S}-\mathrm{S}-\mathrm{CH}_{3}$ have been measured in the frequency range from 5.5 to $34 \mathrm{kmc} / \mathrm{sec}$. From the six rotational constants an $r_{0}$-structure has been calculated. STARK-effect measurements have been made for the $1_{01}-1_{10}$ and $2_{02}-2_{11}$ rotational transitions of $\mathrm{CH}_{3}-\mathrm{S}-\mathrm{S}-\mathrm{CH}_{3}$. The dipole moment was calculated to be (1.985 \pm 0.01$)$ Debye. An approximate value for the barrier to internal rotation of the two methyl tops is given, $V_{3}=(1.6 \pm 0.1) \mathrm{kcal}$. The calculation has been based on triplet splittings of the rotational lines using second order perturbation theory in the torsional wavefunctions and neglecting first order and cross terms in angular momentum.
\end{abstract}

Mikrowellenspektroskopisch ist das Dimethyldisulfid besonders deshalb interessant, weil neben der zu einer Feinstruktur des Spektrums führenden molekülinternen Rotation der Methylgruppen eine niederfrequente Torsionsschwingung in stärkere Wechselwirkung mit der Gesamtrotation des Moleküls treten kann. Es handelt sich um die den Diederwinkel am Schwefel ändernde Torsionsschwingung um die $\mathrm{S}-\mathrm{S}$-Bindung $\left(\bar{v}=116 \mathrm{~cm}^{-1}\right)^{1}$. In der vorliegenden Arbeit wird über die Zuordnung des Spektrums, eine $r_{0}$-Struktur, die Dipolmomentbestimmung und eine näherungsweise Abschätzung des Hinderungspotentials berichtet.

\section{Zuordnung}

Die Zuordnung des sehr linienreichen b-Dipolspektrums gelang schrittweise. Zunächst wurden die Spektren der beiden Molekülisotope

$$
\mathrm{CD}_{3}-\mathrm{S}-\mathrm{S}-\mathrm{CD}_{3} \text { und } \mathrm{CH}_{3}-\mathrm{S}-\mathrm{S}-\mathrm{CH}_{3}
$$

im Frequenzbereich zwischen 5,5 und $34 \mathrm{GHz}$ registriert. Zur Aufnahme der Spektren wurde ein mit $32 \mathrm{kHz}$ STARK-Effekt-modulierter und frequenzstabilisierter Spektrograph benutzt, der schon an anderer Stelle beschrieben worden ist ${ }^{2}$. Es zeigte sich, daß als Folge der Kopplung zwischen Gesamtrotation und interner Rotation der beiden Methylgruppen die meisten Linien des nichtdeuterierten Isotops in engabständige Tripletts oder Quartetts aufgespalten sind. Die Aufspaltung beträgt im allgemeinen zwischen 0,5 und $1 \mathrm{MHz}$, so daß die ineinander verlau-

1 D. W. Scott, H. L. Finke, M. E. Gross, G. B. Guthrie u. H. M. Hurfmann, J. Amer. Chem. Soc. 72, 2424 [1950]. fenden Stark-Effekt-Muster der Multiplettkomponenten als Zuordnungshilfsmittel weitgehend ausschieden. Beim volldeuterierten Isotop dagegen tritt, wegen des größeren Trägheitsmoments der $\mathrm{CD}_{3}$-Gruppe, die Multiplettaufspaltung im Spektrum nicht mehr in Erscheinung und die SтаRк-Effekt-Muster isoliert liegender Linien lassen sich gut auflösen.

Durch Vergleich mit einem aus einer hypothetischen Näherungsstruktur berechneten Modellspektrum und auf Grund ihres STARK-Effektes ließen sich die $\Delta J=0$-Übergänge des deuterierten Moleküls zuordnen. Die etwa um den Faktor 10 intensitätsschwächeren $\Delta J=1$-Übergänge konnten jedoch, da sie meist in ziemlich linienreiche Frequenzbereiche fallen, nicht identifiziert werden. Immerhin ließ sich mit der aus den $Q$-Übergängen gewonnenen Teilinformation, $(A-C)$ und $\varkappa$, die Näherungsstruktur des Moleküls verbessern. Hierzu wurden von den sechs wesentlichen Strukturparametern vier, nämlich die Abstände $r(\mathrm{~S}-\mathrm{S}), r(\mathrm{~S}-\mathrm{C}), r(\mathrm{C}-\mathrm{H})$ und der Winkel $\Varangle(\mathrm{SCH})$ fest vorgegeben und die restlichen zwei, die Winkel $\varphi=\Varangle$ (SSC) und $\delta$ (Diederwinkel), die am unsichersten schienen, iterativ nach dem Gleichungssystem (1) an die experimentellen Werte von $(A-C)$ und $\varkappa$ angepaßt.

$$
\begin{aligned}
& (A-C)_{\exp }-\left.(A-C)\right|_{\varphi_{0}, \delta_{0}} \\
& =\left.\frac{\partial(A-C)}{\partial \varphi}\right|_{\varphi_{0}, \delta_{0}} \cdot \Delta \varphi+\left.\frac{\partial(A-C)}{\partial \delta}\right|_{\varphi_{0}, \delta_{0}} \cdot \Delta \delta, \\
& \varkappa_{\exp }-\left.\varkappa\right|_{\varphi_{0}, \delta_{0}}=\left.\frac{\partial \varkappa}{\partial \varphi}\right|_{\varphi_{0}, \delta_{0}} \cdot \Delta \varphi+\left.\frac{\partial \varkappa}{\partial \delta}\right|_{\varphi_{0}, \delta_{0}} \cdot \Delta \delta .
\end{aligned}
$$

2 H. D. Rudolph, Z. Angew. Phys. 13, 401 [1961]. 
Die auf den rechten Seiten stehenden Differentialquotienten werden im Rechenprogramm durch Differenzenquotienten approximiert, und die verbesserten Strukturparameter $\varphi_{1}=\varphi_{0}+\Delta \varphi$ und $\delta_{1}=\delta_{0}+\Delta \delta$ können als Ausgangswerte für eine weitere Iteration verwendet werden.

Aus der in den Winkeln $\varphi$ und $\delta$ verbesserten Näherungstruktur wurden die Rotationskonstanten und verbesserte Modellspektren neu berechnet. Zwar schlug die Suche nach den $\Delta J=1$-Übergängen bei beiden Molekülisotopen wieder fehl, aber die $Q$ Linien von $\mathrm{CH}_{3}-\mathrm{S}-\mathrm{S}-\mathrm{CH}_{3}$ ließen sich aus ihrer relativen Frequenzlage und der Richtung des $\mathrm{Ab}$ wanderns der STARK-Effekt-Komponenten identifizieren. Die so gewonnene Zusatzinformation, $(A-C)$ und $\varkappa$ auch für $\mathrm{CH}_{3}-\mathrm{S}-\mathrm{S}-\mathrm{CH}_{3}$, gestattete nach demselben Verfahren wie oben eine weitere Verbesserung der Näherungsstruktur durch gleichzeitige Anpassung von vier Strukturparametern, nämlich $\Varangle(\mathrm{SSC}), \delta, r(\mathrm{~S}-\mathrm{S})$ und $r(\mathrm{~S}-\mathrm{C})$. Das soweit ver-

\begin{tabular}{|c|c|c|c|c|c|}
\hline $\begin{array}{c}\text { Rotations- } \\
\text { übergang } \\
I_{K-K_{+}}-I_{K-K_{+}}\end{array}$ & $\begin{array}{l}\text { gemessene } \\
\text { Frequenzen } \\
\text { in GHz }\end{array}$ & $\begin{array}{c}\text { Rotations- } \\
\text { übergang } \\
I_{K-K_{+}}-I_{K-K_{+}}\end{array}$ & $\begin{array}{l}\text { gemessene } \\
\text { Frequenzen } \\
\text { in } \mathrm{GHz}\end{array}$ & $\begin{array}{c}\text { Rotations- } \\
\text { übergang } \\
I_{K-K_{+}}-I_{K^{k 2} K_{+}}\end{array}$ & $\begin{array}{l}\text { gemessene } \\
\text { Frequenzen } \\
\text { in GHz }\end{array}$ \\
\hline \multicolumn{2}{|c|}{$\mathrm{CD}_{3}-\mathrm{S}-\mathrm{S}-\mathrm{CD}_{3}$} & $5_{15}-6_{06}$ & 28,182863 & $9_{27}-9_{18}$ & $\begin{array}{l}13,703721 \\
13,703917\end{array}$ \\
\hline $3_{13}-4_{04}$ & $14,472721 \mathrm{r}$ & \multirow{3}{*}{$6_{16}-7_{07}$} & 28,183177 & \multirow{3}{*}{$10_{28}-10_{19}$} & 13,704116 \\
\hline $202-3_{13}$ & $17,522306 \mathrm{r}$ & & 33,932064 & & 13,762959 \\
\hline $4_{14}-5_{05}$ & 19,356930 & & $33,932268 \mathrm{v}$ & & $13,763213 \mathrm{v}$ \\
\hline $\begin{array}{l}3_{03}-4_{14} \\
5_{15}-6_{06}\end{array}$ & $21,682128 \mathrm{r}$ & \multirow{6}{*}{$\begin{array}{l}2_{11}-3_{22} \\
1_{01}-1_{10} \\
2_{02}-2_{11} \\
3_{03}-3_{12} \\
4_{04}-4_{13}\end{array}$} & 33,932472 & \multirow{3}{*}{$11_{29}-11_{1,10}$} & 13,763467 \\
\hline $\begin{array}{l}5_{15}-6_{06} \\
4_{04}-5_{15}\end{array}$ & $\begin{array}{l}24,253489 \\
25,761909\end{array}$ & & $\begin{array}{r}32,201762 \mathrm{r} \\
5,593376 \mathrm{r}\end{array}$ & & $\begin{array}{l}14,038229 \\
14,038556 \mathrm{v}\end{array}$ \\
\hline $\begin{array}{l}x_{11}-3_{22} \\
2_{10}\end{array}$ & $26,700509 \mathrm{r}$ & & $5,847726 \mathrm{r}$ & & 14,038883 \\
\hline $22_{12}-3_{21}$ & $27,409588 \mathrm{r}$ & & $6,244661 \mathrm{r}$ & $12_{2,10}-12_{1,11}$ & 14,557285 \\
\hline $5_{05}-6_{16}$ & 29,784428 & & 6,801828 & & $14,557693 \mathrm{v}$ \\
\hline $4_{04}-4_{13}$ & $5,638280 \mathrm{r}$ & & 6,801994 & \multirow{4}{*}{$13_{2,11}-13_{1,12}$} & 14,558101 \\
\hline $5_{05}-5_{14}$ & 6,319495 & \multirow{4}{*}{$5_{05}-5_{14}$} & 6,802156 & & 15,343220 \\
\hline $6_{06}-6_{15}$ & 7,194744 & & 7,541688 & & 15,343728 \\
\hline $8_{08}-8_{17}$ & 9,602190 & & 7,541912 & & 15,344248 \\
\hline $3_{21}-3_{12}$ & 12,651734 & & 7,542120 & \multirow{4}{*}{$5_{15}-5_{24}$} & 18,286350 \\
\hline $4_{22}-4_{13}$ & 12,301062 & \multirow{3}{*}{$7_{07}-7_{16}$} & 9,665200 & & 18,286660 \\
\hline $5_{23}-5_{14}$ & 11,929518 & & $9,665570 \mathrm{v}$ & & 18,286960 \\
\hline $6_{24}-6_{15}$ & 11,578449 & & 9,665940 & & 18,287440 \\
\hline $7_{25}-7_{16}$ & 11,292192 & \multirow{3}{*}{$8_{08}-8_{17}$} & 11,089134 & \multirow[t]{4}{*}{$6_{16}-6_{25}$} & 19,048810 \\
\hline $3_{13}-3_{22}$ & $13,944222 \mathrm{r}$ & & $11,089602 \mathrm{v}$ & & 19,048927 \\
\hline $4_{14}-4_{23}$ & $14,398775 \mathrm{r}$ & & 11,090070 & & 19,049345 \\
\hline $5_{15}-5_{24}$ & 14,970846 & \multirow[t]{3}{*}{$9_{09}-9_{18}$} & 12,767622 & & 19,049828 \\
\hline $4_{22}-4_{31}$ & $22,023448 \mathrm{r}$ & & 12,768204 & \multirow[t]{3}{*}{$7_{17}-7_{26}$} & 19,943628 \\
\hline $5_{23}-5_{32}$ & 21,909205 & & 12,768774 & & 19,944174 \\
\hline $6_{24}-6_{33}$ & 21,718146 & \multirow[t]{3}{*}{$10_{0,10}-10_{19}$} & 14,695216 & & 19,944695 \\
\hline $7_{25}-7_{34}$ & 21,431844 & & $14,695872 \mathrm{v}$ & \multirow[t]{3}{*}{$8_{18}-8_{27}$} & 20,971334 \\
\hline $8_{26}-8_{35}$ & 21,039781 & & 14,696577 & & 20,971901 \\
\hline $4_{32}-4_{23}$ & $22,146964 \mathrm{r}$ & \multirow{3}{*}{$11_{0,11}-11_{1,10}$} & 16,852796 & & 20,972475 \\
\hline $5_{33}-5_{24}$ & 22,192578 & & 16,853615 & \multirow[t]{4}{*}{$10_{1,10}-10_{29}$} & 23,421242 \\
\hline & & & 16,854425 & & 23,421326 \\
\hline & & \multirow[t]{3}{*}{$12_{0,12}-12_{1,11}$} & 19,209415 & & 23,422008 \\
\hline \multirow{3}{*}{\multicolumn{2}{|c|}{$\mathbf{C H}_{3}-\mathrm{S}-\mathrm{S}-\mathbf{C H}_{3}$}} & & $\begin{array}{l}19,209615 \\
19,210427\end{array}$ & & $\begin{array}{l}23,422741 \\
24,837787\end{array}$ \\
\hline & & & 19,211336 & \multirow{3}{*}{$11_{1,11}-11_{2,10}$} & 24,837925 \\
\hline & & \multirow[t]{2}{*}{$14_{0,14}-14_{1,13}$} & 24,356319 & & 24,838690 \\
\hline & & & 24356912 & & 24,839515 \\
\hline $\begin{array}{l}0_{00}-1_{11} \\
2_{02}-3_{13}\end{array}$ & $\begin{array}{l}10,734168 \mathrm{r} \\
20,895948 \mathrm{r}\end{array}$ & \multirow{4}{*}{$7_{25}-7_{16}$} & $\begin{array}{l}24,357725 \\
24,358937\end{array}$ & \multirow{4}{*}{$\begin{array}{c}9_{27}-9_{36} \\
10_{28}-10_{37} \\
11_{29}-11_{38}\end{array}$} & $\begin{array}{l}25,732056 \\
25,100864\end{array}$ \\
\hline $3_{03}-4_{14}$ & 25,807519 & & $?$ & & 24,384425 \\
\hline & 25,807636 & & 14,095966 & & 24,384746 \\
\hline & 25,807779 & & 14,096135 & & 24,384890 \\
\hline $2_{12}-3_{03}$ & $11,017832 \mathrm{r}$ & $8_{26}-8_{17}$ & 13,827555 & $12_{2,10}-12_{39}$ & 23,620125 \\
\hline $3_{13}-4_{04}$ & $16,696232 \mathrm{r}$ & & $13,827718 \mathrm{v}$ & & 23,620345 \\
\hline $4_{14}-5_{05}$ & 22,427516 & & 13,827882 & & 23,620450 \\
\hline
\end{tabular}

Tab. 1. Die in den Linientabellen mit „r“ gekennzeichneten Übergänge wurden zur Berechnung der Rotationskonstanten verwendet, die mit „ “" gekennzeichneten Triplettübergänge zur Berechnung des Hinderungspotentials der internen Rotation. "Absoluter Meßfehler ca. $50 \mathrm{kHz}$, relativer Meßfehler innerhalb eines Multipletts ca. $5 \mathrm{kHz}$. 
besserte Molekülmodell machte endlich eine ausreichend genaue Vorausberechnung der $\Delta J=1$-Übergänge möglich, so daß auch diese Linien im Spektrum identifiziert werden konnten.

\section{Struktur}

Die sechs Rotationskonstanten (Tab. 2), drei für jedes Molekülisotop, wurden dazu benutzt, eine $r_{0}$ Struktur ${ }^{3}$ zu bestimmen. Das Ergebnis dieser Rechnung ist in Tab. 3 zusammengefaßt. In die Rechnung gehen zwei Voraussetzungen ein. Es wird angenommen, die Methylgruppen besäßen $C_{3 \mathrm{v}}$-Symmetrie und ihre Symmetrie- und Drehachse falle mit der S-C. Bindungsachse zusammen. Beide Annahmen werden nur näherungsweise erfüllt sein. Aus einer genaueren Analyse der Feinstruktur des Rotationsspektrums von $\mathrm{CH}_{3}-\mathrm{S}-\mathrm{CH}_{3}{ }^{4,5}$ hat sich z. B. für dieses, dem Dimethyldisulfid verwandte Molekül, ein leichtes Abknicken der Symmetrieachsen um ca. $2^{\circ}$

\begin{tabular}{|ll|c|c|}
\hline & & $\mathrm{CH}_{3}-\mathrm{S}-\mathrm{S}-\mathrm{CH}_{3}$ & $\mathrm{CD}_{3}-\mathrm{S}-\mathrm{S}-\mathrm{CD}_{3}$ \\
\hline$\varkappa$ & & $-0,912020$ & $-0,901913$ \\
$A$ & $\mathrm{GHz}$ & 8,16364 & 6,71760 \\
$B$ & $\mathrm{GHz}$ & 2,81642 & 2,40492 \\
$C$ & $\mathrm{GHz}$ & 2,57037 & 2,18250 \\
$I_{a}$ & $\mathrm{AMU} \cdot \AA^{2}$ & 61,9256 & 75,2557 \\
$I_{b}$ & $\mathrm{AMU} \cdot \AA^{2}$ & 179,497 & 210,210 \\
$I_{c}$ & $\mathrm{AMU} \cdot \AA^{2}$ & 196,679 & 231,632 \\
\hline
\end{tabular}

Tab. 2. Rotationskonstanten von Dimethyldisulfid und Dimethyldisulfid- $\mathrm{d}_{6}$. Die zur Berechnung der Rotationskonstanten verwendeten Ubergänge sind in den Linientabellen mit $r$ gekennzeichnet. Genauigkeit der Rotationskonstanten: \pm 10 $\mathrm{kHz}$, Umrechnungsfaktor: $A \cdot I_{a}=505,538 \mathrm{GHz} \cdot \mathrm{AMU} \cdot \AA^{2}$.

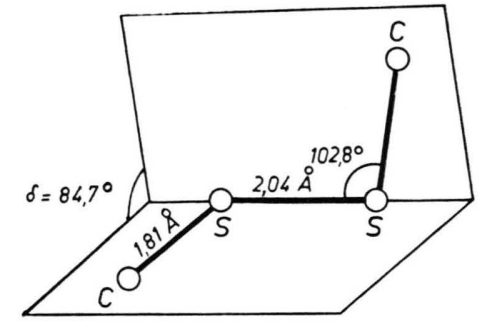

Abb. 1. Geometrische Struktur des Molekülrumpfes von Dimethyldisulfid. Die $b$-Achse des Trägheitsellipsoids fällt mit $\operatorname{der} C_{2}$-Symmetrieachse zusammen. Die $a$-Achse verläuft annähernd parallel zur S-S-Bindung.

3 C. C. Costain, J. Chem. Phys. 29, 864 [1958].

${ }^{4}$ L. Pierce u. M. Hayashi, J. Chem. Phys. 35, 479 [1961].

${ }^{5}$ H. Dreizler u. H. D. Rudolph, Z. Naturforschg. 17 a, 712 [1962].

6 H. Dreizler, G. Dendl u. W. Feder, paper Nr. 210, presented at the $8^{\text {th }}$ European Congress on Molecular Spectroscopy, Copenhagen 1965.

\begin{tabular}{|c|c|c|}
\hline $\begin{array}{l}r(\mathrm{~S}-\mathrm{S})=2,03_{8} \AA \\
r(\mathrm{~S}-\mathrm{C})=1,81_{0} \AA \\
r(\mathrm{C}-\mathrm{H})=1,09_{7} \AA\end{array}$ & \multicolumn{2}{|c|}{$\begin{array}{r}\Varangle(\mathrm{SSC})=102,8^{0} \\
\Varangle(\mathrm{SCH})=108,9^{0} \\
\delta=84,7^{0} *\end{array}$} \\
\hline$\lambda_{a}= \pm 0,485$ & $\lambda_{b}=0,721$ & $\lambda_{c}= \pm 0,495$ \\
\hline \multicolumn{3}{|c|}{$\begin{array}{l}\left( \pm \lambda_{a}, \lambda_{b}, \pm \lambda_{c}\right)=\text { Richtungscosinus der Symmetrie- } \\
\text { und Drehachsen der Methylgruppen bezüglich der } \\
\text { Hauptträgheitsachsen. } \\
\text { Bei } \mathrm{F}-\mathrm{S}-\mathrm{S}-\mathrm{F}^{7} \text { beträgt der Diederwinkel } \delta= \\
87,9 \pm 1,5^{0} \text {, bei faserförmigem Schwefel } 87^{0} 8 \text {. }\end{array}$} \\
\hline
\end{tabular}

Tab. 3. $r_{0}$-Strukturparameter von Dimethyldisulfid.

nach außen ergeben und beim Dimethylsulfoxyd ${ }^{6}$ ergab eine $r_{\mathrm{s}}$-Strukturrechnung eine leichte Asymmetrie der Methylgruppen.

\section{Dipolmoment}

Das Dipolmoment wurde aus dem Stark-Effekt der beiden Übergänge $1_{01}-l_{10}$ und $2_{02}-2_{11}$ von $\mathrm{CH}_{3}-\mathrm{S}-\mathrm{S}-\mathrm{CH}_{3}$ bestimmt. Diese Übergänge zeigen noch keine Multiplettaufspaltung durch die Methylrotation. Der Spektrograph war für diese Messungen mit dem Übergang $J=1-2$ von OSC bei $24,325925 \mathrm{GHz}$ geeicht worden ${ }^{9}$. Zur Berechnung von $\mu_{b}$ wurde die Matrix des Hamilton-Operators

$$
H=A P_{a}^{2}+B P_{b}^{2}+C P_{c}^{2}+\mu_{b} E \cos (b Z)
$$

in der Basis des symmetrischen Kreisels aufgestellt. Die Energieniveaus $W_{J, K-K+, M}$ wurden näherungsweise berechnet, indem aus der unendlichen Matrix

\begin{tabular}{|c|c|c|c|c|}
\hline Ùbergang & $M$ & $\begin{array}{c}E \\
\mathrm{~V} / \mathrm{cm}\end{array}$ & $\begin{array}{c}\Delta v \\
\mathrm{MHz}\end{array}$ & $\begin{array}{c}\mu_{b} \text { (ber.) } \\
\text { Debye }\end{array}$ \\
\hline $\begin{array}{l}1_{01}-1_{10} \\
2_{02}-2_{11}\end{array}$ & 1 & $\begin{array}{l}179,7 \\
224,1 \\
269,5 \\
314,4 \\
179,2 \\
224,6 \\
269,5 \\
314,4\end{array}$ & $\begin{array}{r}3,00 \\
4,69 \\
6,75 \\
9,19 \\
3,41 \\
5,34 \\
7,75 \\
10,45\end{array}$ & $\begin{array}{l}1,98_{3} \\
1,98_{6} \\
1,98_{3} \\
1,98_{2} \\
1,98_{8} \\
1,98_{4} \\
1,99_{2} \\
1,98_{2}\end{array}$ \\
\hline
\end{tabular}

Tab. 4. Dipolmoment von $\mathrm{CH}_{3}-\mathrm{S}-\mathrm{S}-\mathrm{CH}_{3}$.

7 R. L. Kuсzкоwsкi, Diss. Abstr. 25, 64-11, 561.

8 J. A. Prins, J. Schenk u. L. H. J. W Achters, Physica 23, 746 [1957].

9 S. A. Marschall u. J. Weber, Phys. Rev. 105, 1502 [1957]. 
die durch $J$ und $J+2$ begrenzte Submatrix herausgeschnitten und nach dem Verfahren von JAcoBI diagonalisiert wurde. (In beiden Fällen wurde nur die Frequenzablage des $M=J$-Satelliten gemessen; für $M=J$ beginnt die Energiematrix mit der Submatrix J.) Das Ergebnis ist in Tab. 4 zusammengefaßt.

\section{Hinderungspotential}

Die Multiplettaufspaltung der Rotationslinien von $\mathrm{CH}_{3}-\mathrm{S}-\mathrm{S}-\mathrm{CH}_{3}$ gestattet die Berechnung des Hinderungspotentials $V_{3}$ der internen Methylrotation.

Hier soll nur eine relativ grobe Abschätzung von $V_{3}$ im Rahmen einer vereinfachten Störungsrech- nung zweiter Ordnung, die auf der Triplettaufspaltung der Rotationslinien basiert, gegeben werden. Da eine genauere Berechnung von $V_{3}$ in einer späteren Arbeit beabsichtigt ist, sei die Theorie etwas ausführlicher dargestellt. Wir haben die Wechselwirkung mit der S-S-Torsion vernachlässigt und das reale Molekül ersetzt durch das Modell eines starren Molekülrumpfes mit zwei behindert drehbaren, in sich starren symmetrischen Teilkreiseln, den Methylgruppen. Für dieses Model lautet der HamiltonOperator unter Annahme eines reinen dreizähligen Cosinuspotentials für die interne Rotation $\left(V_{12}\right.$, $V_{12}{ }^{\prime}$ etc. vernachlässigt) ${ }^{10}$ :

$$
\begin{aligned}
H= & \left(A+2 F_{-} \alpha^{2}\right) P_{a}^{2}+\left(B+2 F_{+} \beta^{2}\right) P_{b}^{2}+\left(C+2 F_{-} \gamma^{2}\right) P_{c}^{2}+2 F_{-} \alpha \gamma\left(P_{a} P_{c}+P_{c} P_{a}\right) \\
& -2 F_{-} \alpha\left(p_{1}-p_{2}\right) P_{a}-2 F_{+} \beta\left(p_{1}+p_{2}\right) P_{b}-2 F_{-} \gamma\left(p_{1}-p_{2}\right) P_{c} \\
& +F p_{1}^{2}+\frac{1}{2} V_{3}\left(1-\cos 3 \alpha_{1}\right)+F p_{2}^{2}+\frac{1}{2} V_{3}\left(1-\cos 3 \alpha_{2}\right)+F^{\prime}\left(p_{1} p_{2}+p_{2} p_{1}\right)
\end{aligned}
$$

\section{Bezeichnungen:}

$A, B, C=$ Rotationskonstanten;

$\alpha=\lambda_{a} I_{\alpha} / I_{a} ; \quad \beta=\lambda_{b} I_{\alpha} / I_{b} ; \quad \gamma=\lambda_{c} I_{\alpha} / I_{c} ;$

$\pm \lambda_{a}, \lambda_{b}, \pm \lambda_{c}$ Richtungscosinus der Symmetrie- und Drehachsen der Teilkreisel bezüglich der Hauptträgheitsachsen;

$I_{a} \quad=$ Trägheitsmoment einer Methylgruppe;

$I_{a}, I_{b}, I_{c} \quad$ Hauptträgheitsmomente des Gesamtmoleküls;

$P_{a}, P_{b}, P_{c}=$ Drehimpulskomponenten um die Hauptträgheitsachsen;

$p_{i} \quad=$ Gesamtdrehimpuls des $i$-ten Teilkreisels um seine Symmetrieachse $(i=1,2)$;

$a_{i} \quad=$ Drehwinkel des $i$-ten Teilkreisels relativ zum Molekülrumpf;

$$
\begin{gathered}
F_{+}=\frac{h^{2}}{8 \pi^{2} I_{\alpha}} \frac{1}{r+q} ; \quad F_{-}=\frac{h^{2}}{8 \pi^{2} I_{a}} \frac{1}{r-q} ; \\
F^{\prime}=-\frac{h^{2}}{8 \pi^{2} I_{\alpha}} \frac{q}{\left(r^{2}-q^{2}\right)} ; \quad F=\frac{h^{2}}{8 \pi^{2} I_{a}} \frac{r}{\left(r^{2}-q^{2}\right)} ; \\
r=1-\lambda_{a}^{2}\left(I_{\alpha} / I_{a}\right)-\lambda_{b}{ }^{2}\left(I_{\alpha} / I_{b}\right)-\lambda_{c}^{2}\left(I_{\alpha} / I_{c}\right) ; \\
q=\lambda_{a}^{2}\left(I_{\alpha} / I_{a}\right)-\lambda_{b}{ }^{2}\left(I_{\alpha} / I_{b}\right)+\lambda_{c}^{2}\left(I_{a} / I_{c}\right) .
\end{gathered}
$$

Die Symmetriegruppe dieses Operators läßt sich als direktes Produkt zweier Untergruppen schreiben ${ }^{11}$ :

$$
C_{3 \mathrm{v}}{ }^{-} \times C_{3}{ }^{+} \text {. }
$$

Das Eigenwertproblem wird näherungsweise gelöst. Hierzu wird die Wechselwirkung zwischen interner Rotation und Gesamtrotation des Moleküls [zweite Zeile von (2)] als Störung behandelt und

10 L. Pierce, J. Chem. Phys. 34, 498 [1961]. die Energiematrix wird aufgestellt in der Basis des Funktionensystems

$$
S_{J K M}(\varphi, \vartheta, \chi) \cdot U_{v_{1} \sigma_{1}}\left(\alpha_{1}\right) \cdot U_{v_{2} \sigma_{2}}\left(\alpha_{2}\right),
$$

wobei die $S_{J K M}$ die Eigenfunktionen des starren symmetrischen Kreisels, die $U_{v_{i} \sigma_{i}}$ (periodische $\mathrm{MA}_{\mathrm{A}}$ THIEU-Funktionen) die Eigenfunktionen der reinen Torsionsenergie:

$$
H_{\mathrm{tors}, i}=F \cdot p_{i}{ }^{2}+\frac{1}{2} V_{3}\left(1-\cos 3 \alpha_{i}\right) \quad(i=1,2)
$$

\begin{tabular}{|c|c|c|c|c|}
\hline Spezies & $A_{1} A$ & $E E_{a}+E E_{b}$ & $E A$ & $A_{1} E_{a}+A_{1} E_{b}$ \\
\hline$\sigma_{1}, \sigma_{2}$ & 0,0 & $\begin{array}{rr}0, & 1 \\
0, & -1 \\
1, & 0 \\
-1, & 0\end{array}$ & $\begin{array}{rr}1, & -1 \\
-1, & 1\end{array}$ & $\begin{array}{rr}1, & 1 \\
-1, & -1\end{array}$ \\
\hline
\end{tabular}

sind.

Für $v_{1}=v_{2}=0$ erhält man Torsionseigenfunktionen, die den in Tab. 5 angegebenen, durch die jeweilige $\left(\sigma_{1}, \sigma_{2}\right)$-Kombination gekennzeichneten Symmetriespezies von $\mathrm{C}_{3 \mathrm{v}}{ }^{-} \times \mathrm{C}_{3}{ }^{+}$angehören. (Im Falle hohen Hinderungspotentials sind die dazugehörigen Torsionsenergien nahezu entartet. Beim $\mathrm{CH}_{3}-\mathrm{S}-\mathrm{S}$ $-\mathrm{CH}_{3}$ beträgt ihre Aufspaltung nur ca. $60 \mathrm{MHz}$.)

Tab. 5. Symmetriespezies der Torsionseigenfunktionen im Torsionsgrundzustand $\left(v_{1}=v_{2}=0\right)$ bei $C_{3 \mathrm{v}^{-}} \times C_{3}{ }^{+}$-Symmetrie des Hamilton-Operators.

Auf den vier Torsionsniveaus bauen sich jeweils die Termschemata der Rotationsniveaus auf, die infolge der Wechselwirkung mit „ihrem“ Torsionszustand

11 H. Dreizler, Z. Naturforschg. 16 a, 477, 1354 [1961]. 
von Spezies zu Spezies etwas unterschiedliche Termabstände haben. Dementsprechend fallen auch die Frequenzen des gleichen Rotationsübergangs für die verschiedenen Spezies nicht zusammen und im Spektrum erscheint der Übergang in ein Multiplett aufgespalten.

In der oben gewählten Basis ist die Energiematrix diagonal in $\sigma_{1}, \sigma_{2}, J$ und $M$, nichtdiagonal in $v_{1}, v_{2}$ und $K$. Auf Grund der Diagonalität in $\sigma_{1}$ und $\sigma_{2}$ kann demnach die Störungsrechnung für die verschiedenen Spezies getrennt durchgeführt werden. Da der Abstand der Torsionsniveaus innerhalb einer Spezies im Vergleich zu dem der Rotationsniveaus groß ist, haben wir die Störungsrechnung in $v_{1}$ und $v_{2}$ mittels einer einmalig angewandten vaN $\mathrm{V}_{\text {LECK }}$ Transformation ${ }^{12}$ nur bis zur zweiten Ordnung durchgeführt. Als „effektiver Hamilton-Operator“ der Rotation ergibt sich dann:

$$
\begin{aligned}
H_{\text {eff }}=[A & \left.+2 F_{-} \alpha^{2}+4 F_{-}{ }^{2} \alpha^{2}\left(\left(\frac{p p}{\Delta}\right)_{1}+\left(\frac{p p}{\Delta}\right)_{2}\right)\right] P_{a}{ }^{2}+\left[B+2 F_{+} \beta^{2}+4 F_{+}{ }^{2} \beta^{2}\left(\left(\frac{p p}{\Delta}\right)_{1}+\left(\frac{p p}{\Delta}\right)_{2}\right)\right] P_{b}{ }^{2} \\
& +\left[C+2 F_{-} \gamma^{2}+4 F_{-}{ }^{2} \gamma^{2}\left(\left(\frac{p p}{\Delta}\right)_{1}+\left(\frac{p p}{\Delta}\right)_{2}\right)\right] P_{c}{ }^{2} \\
& +\left[2 F_{-} \alpha \gamma+4 F_{-}{ }^{2} \alpha \gamma\left(\left(\frac{p p}{\Delta}\right)_{1}+\left(\frac{p p}{\Delta}\right)_{2}\right)\right]\left(P_{a} P_{c}+P_{c} P_{a}\right)-8 \alpha F_{-} F^{\prime}\left(\left(\frac{p p}{\Delta}\right)_{1} p_{2}-\left(\frac{p p}{\Delta}\right)_{2} p_{1}\right) P_{a} \\
& +4 F_{-} F_{+} \alpha \beta\left(\left(\frac{p p}{\Delta}\right)_{1}-\left(\frac{p p}{\Delta}\right)_{2}\right)\left(P_{a} P_{b}+P_{b} P_{a}\right)-8 \beta F_{+} F^{\prime}\left(\left(\frac{p p}{\Delta}\right)_{1} p_{2}+\left(\frac{p p}{\Delta}\right)_{2} p_{1}\right) P_{b} \\
& +4 F_{-} F_{+} \beta \gamma\left(\left(\frac{p p}{\Delta}\right)_{1}-\left(\frac{p p}{\Delta}\right)_{2}\right)\left(P_{b} P_{c}+P_{c} P_{b}\right)-8 \gamma F_{-} F^{\prime}\left(\left(\frac{p p}{\Delta}\right)_{1} p_{2}-\left(\frac{p p}{\Delta}\right)_{2} p_{1}\right) P_{c} \\
& -2 F_{-} \alpha\left(p_{1}-p_{2}\right) P_{a}-2 F_{+} \beta\left(p_{1}+p_{2}\right) P_{b}-2 F_{-} \gamma\left(p_{1}-p_{2}\right) P_{c}+F^{\prime}\left(p_{1} p_{2}+p_{2} p_{1}\right)
\end{aligned}
$$

Bezeichnungen:

$$
\begin{aligned}
\left(\frac{p p}{\Delta}\right)_{i}= & \sum_{\substack{v_{i}^{\prime} \\
v_{i}^{\prime} v_{i}}} \frac{\left|\left(v_{i} \sigma_{i}\left|p_{i}\right| v_{i}^{\prime} \sigma_{i}\right)\right|^{2}}{E_{v_{i} \sigma_{i}}-E_{v_{i}{ }^{\prime} \sigma_{i}}} \quad(i=1,2) \\
E_{v_{i} \sigma_{i}}= & \begin{array}{l}
\text { Energieeigenwert des } \\
\text { des } i \text {-ten Teilkreisels; }
\end{array} \\
p_{i} & =\left(v_{i} \sigma_{i}\left|p_{i}\right| v_{i} \sigma_{i}\right) .
\end{aligned}
$$

Bei der Auswertung der Triplettaufspaltungen wurden nur die beiden ersten Zeilen von (3) berücksichtigt, d. h. die in den Drehimpulskomponenten linearen und gemischt quadratischen Glieder wurden vernachlässigt, weil sie in 1 . Ordnung keine Beiträge zu $H_{\text {eff }}$ liefern. Die in (3 a) auftretenden Störsummen sind vom Einkreiselproblem her bekannt und liegen in Tabellenform vor ${ }^{13,14}$.

Man erhält für die verschiedenen, durch die spezielle $\left(\sigma_{1}, \sigma_{2}\right)$-Kombination gekennzeichneten Symmetriespezies jeweils einen Hamilton-Operator vom Typ des starren asymmetrischen Kreisels mit speziesabhängig abgeänderten Rotationskonstanten (vgl. Tab. 6). Bei der Berechnung der Korrekturen wurde

\begin{tabular}{|c|c|c|c|}
\hline Spezies & $A_{1} A$ & $\left(E E_{a}+E E_{b}\right)$ & $\begin{array}{c}\left(A_{1} E_{a}+A_{1} E_{b}\right) \\
\text { und } E A\end{array}$ \\
\hline $\begin{array}{l}\text { Korrek- } \\
\text { turgrößen } \\
\Delta A_{0 \sigma 1,0 \sigma 2} \\
\Delta B_{0 \sigma 1,0 \sigma 2} \\
\Delta C_{0 \sigma 1,0 \sigma 2}\end{array}$ & $\begin{array}{l}2 F \alpha^{2} W_{00}^{(2)} \\
2 F \beta^{2} W_{00}^{(2)} \\
2 F \gamma^{2} W_{00}^{(2)}\end{array}$ & $\begin{array}{l}F \alpha^{2}\left(W_{00}^{(2)}+W_{01}^{(2)}\right) \\
F \beta^{2}\left(W_{00}^{(2)}+W_{01}^{(2)}\right) \\
F \gamma^{2}\left(W_{00}^{(2)}+W_{01}^{(2)}\right)\end{array}$ & $\begin{array}{l}2 F \alpha^{2} W_{01}^{(2)} \\
2 F \beta^{2} W_{01}^{(2)} \\
2 F \gamma^{2} W_{01}^{(2)}\end{array}$ \\
\hline \multicolumn{4}{|c|}{$\begin{array}{l}\text { Abkürzung: } W_{0 \sigma_{i}}^{(2)}=1+4 F \sum \frac{\left|\left(0 \sigma_{i}\left|p_{i}\right| v_{i}^{i} \sigma_{i}\right)\right|^{2}}{E_{0 \sigma_{i}}-E_{v i \sigma_{i}}} \\
\text { Der beim Dimethyldisulfid ca. } 1 \% \text { betragende Unter- } \\
\text { schied zwischen } F_{+} \text {und } F_{-} \text {ist vernachlässigt, d.h. es } \\
\text { ist gesetzt: } F_{-}=F_{+}=F \text {. Für höhere Werte von } \\
s=4 V_{3} /(9 F) \text { gilt } 14 W_{00}^{(2)} \approx-2 W_{01}^{(2)} \text {, was die Aus- } \\
\text { wertung etwas erleichtert. }\end{array}$} \\
\hline
\end{tabular}
der beim Dimethyldisulfid etwa $1 \%$ betragende Unterschied von $F_{-}$und $F_{+}$vernachlässigt, und beide Werte wurden durch ihren Mittelwert $F$ ersetzt.

Die effektiven Rotationskonstanten der $\left(E E_{a}\right.$ $\left.+E E_{b}\right)$-Spezies sind gerade das arithmetische Mit-

12 E. C. Kemble, Quantum Mechanics, Dover Publications, Inc., New York 1958, S. 394.

13 D. R. Herschbach, Tables for the Internal Rotation Problem, Dept. of Chemistry, Harvard University, 1957.

Tab. 6. Korrekturgrößen der Rotationskonstanten (Triplettnäherung) für die verschiedenen Symmetriespezies des Torsionsgrundzustandes $\left(v_{1}=v_{2}=0\right)$.

tel der Rotationskonstanten von $A_{1} A$ und $\left(A_{1} E_{a}\right.$ $\left.+A_{1} E_{b}\right)$ - bzw. $E A$-Spezies. Dementsprechend spalten in dieser Näherung die Rotationslinien in gleichabständige Tripletts auf. Zwei derartige Tripletts, die auch im Intensitätsverhältnis ihrer Komponenten gut der aus der Spinstatistik ${ }^{11}$ gewonnenen Voraussage entsprechen, sind in Abb. 2 gezeigt. Zur Berechnung von $V_{3}$ wurden für die verschiedenen Spezies die effektiven Rotationskonstanten mit einem

14 M. Hayashi u. L. Pierce, Tables for the Internal Rotation Problem, Dept. of Chemistry, University of Notre Dame, Notre Dame, Indiana. 

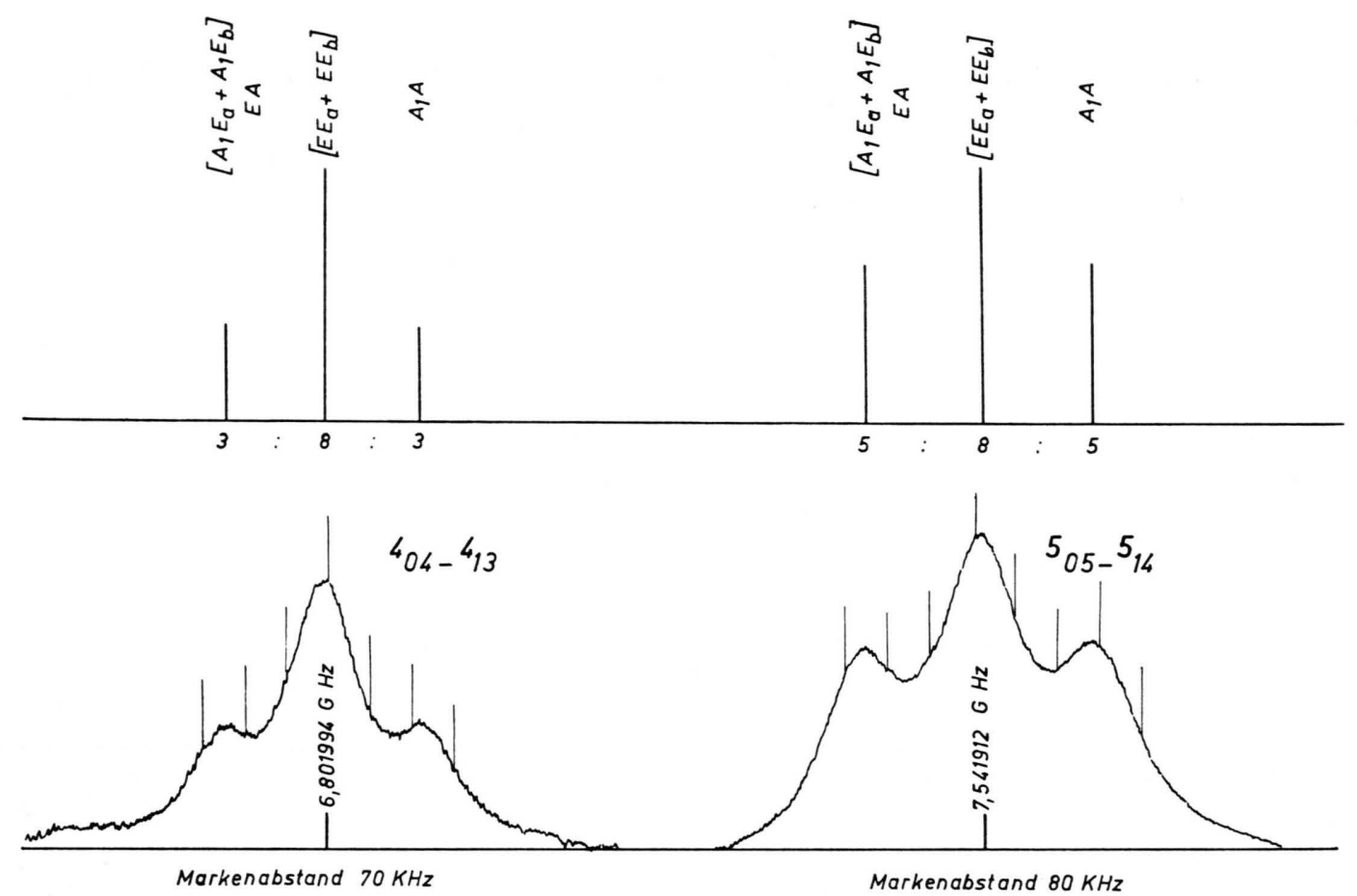

Abb. 2. Triplettaufspaltung beim $\mathrm{CH}_{3}-\mathrm{S}-\mathrm{S}-\mathrm{CH}_{3}$. (Oben das theoretisch auf Grund der Spingewichte vorausgesagte Intensitätsverhältnis. Annahmen: Gleiche Dipolmatrixelemente der verschiedenen Symmetriespezies; $C_{2}$-Konfigurationssymmetrie; $C_{3 \mathrm{v}^{-}}{ }^{-} \times C_{3}{ }^{+}$-Symmetrie des Hamilon-Operators.)

Programm des starren asymmetrischen Rotators berechnet. (Die bei der Anpassungsrechnung verwendeten Triplettübergänge sind in der Linientabelle mit $v$ gekennzeichnet.) Aus den effektiven Rotationskonstanten wurden die Korrekturgrößen und aus diesen nach Tab. 6 die Störsummen bestimmt. Die in die Rechnung eingehenden Geometriefaktoren $(F$, $\alpha, \beta, \gamma)$ wurden aus der $r_{0}$-Struktur übernommen. Interpolation in der Tabelle von Hayashi und Pierce ${ }^{14}$ lieferte die in Tab. 7 angegebenen Werte.

$$
\begin{aligned}
& \text { Geometriefaktoren (übernommen aus der } r_{0} \text {-Struktur): } \\
& \alpha= \pm 0,0256 ; \quad \beta=0,0131 ; \quad \gamma= \pm 0,0082 \text {; } \\
& I_{\alpha}=3,26 \mathrm{AMU} \cdot \AA^{2} ; \quad F=159,1 \mathrm{GHZ}
\end{aligned}
$$

Tab. 7. Hinderungspotential der Methyltorsion.

Die aus den Korrekturgrößen $\Delta A_{0 \sigma_{i}}$ etc. berechneten $V_{3}$-Werte streuen $( \pm 6 \%)$, was offensichtlich an der nahen K-Entartung beim Dimethyldisulfid liegt $(x \approx-0,92)$. Durch sie gewinnen trotz des relativ hohen Hinderungspotentials die in der Triplettnäherung vernachlässigten Teile von (3) an Einfluß. Im Spektrum zeigt sich das am Auftreten von
Quartetts [Aufhebung der Entartung von $\left(A_{1} E_{a}\right.$ $+A_{1} E_{b}$ ) - und $E A$-Spezies] und in "Anomalien“ im Intensitätsverhältnis der Multiplettkomponenten. Ein Rechenprogramm, das ohne obige Vernachlässigungen $V_{3}$ direkt aus (3) zu berechnen gestattet, ist zur Zeit in Arbeit.

Neben der hier untersuchten Form des Dimethyldisulfids, $\mathrm{CH}_{3}-\mathrm{S}-\mathrm{S}-\mathrm{CH}_{3}$, könnte ähnlich wie bei dem von Kuczkowski ${ }^{7}$ untersuchten Difluordisulfid, noch eine zweite isomere Form existieren,

$$
\left(\mathrm{CH}_{3}\right)_{2} \mathrm{~S}=\mathrm{S} \text {. }
$$

Wegen der speziell auf das $\mathrm{CH}_{3}-\mathrm{S}-\mathrm{S}-\mathrm{CH}_{3}$ hinzielenden Darstellung ist jedoch die zweite Form in den von uns untersuchten Proben nicht enthalten. Eine gezielte Darstellung von $\left(\mathrm{CH}_{3}\right)_{2} \mathrm{~S}=\mathrm{S}$ und eine Untersuchung des Mikrowellenspektrums sind geplant.

Unser Dank gilt Herrn Dr. R. Peter und Herrn Dr. H.-G. Schirdewahn, die bei der Erstellung der Rechenprogramme mitarbeiteten, Herrn Dipl.-Chem. H. HARTENSTEIn für die Darstellung der nichtdeuterierten Substanz und der Deutschen Forschungsgemeinschaft, die diese Arbeit durch großzügige Gewährung von Sachmitteln unterstützte. 